\title{
Narrative review of hyperthermic intraperitoneal chemotherapy (HIPEC) in patients with advanced ovarian cancer: a critical reappraisal of the current evidence
}

\author{
John Spiliotis ${ }^{1,2}$, Anastasia Prodromidou ${ }^{3}$ \\ ${ }^{1}$ Athens Medical Centre, Athens, Greece; ${ }^{2}$ European Interbalkan Medical Centre, Thessaloniki, Greece; ${ }^{3}$ Faculty of Medicine, National and \\ Kapodistrian University of Athens, Athens, Greece \\ Contributions: (I) Conception and design: J Spiliotis; (II) Administrative support: J Spiliotis; (III) Provision of study materials or patients: A \\ Prodromidou; (IV) Collection and assembly of data: A Prodromidou; (V) Data analysis and interpretation: J Spiliotis, A Prodromidou; (VI) \\ Manuscript writing: All authors; (VII) Final approval of manuscript: All authors. \\ Correspondence to: Anastasia Prodromidou, MD, PhD. Faculty of Medicine, National and Kapodistrian University of Athens, Timotheou 82-86, \\ 16231, Athens, Greece. Email: a.prodromidou@hotmail.com.
}

\begin{abstract}
The implementation of hyperthermic intraperitoneal chemotherapy (HIPEC) in the management of advanced stage epithelial ovarian cancer (EOC) as a standard practice remains debatable despite the emerging data supporting its beneficial effect when used to supplement cytoreductive procedures. The aim of the present review was an attempt to accumulate the currently available evidence on the use of HIPEC for patients with primary and recurrent EOC and to address directives of future research. Based on the currently available literature, the progress in cytoreductive surgical procedures and chemotherapy has brought significant improvement in the management and survival outcomes of selected patients with advanced EOC. The addition of HIPEC seems encouraging based on the outcomes of high-quality clinical trials. There are significant parameters on the use of CRS and HIPEC such as patient selection, the sequencing of procedures, the type of chemotherapy agent and time and the temperature of hyperthermic procedures which require additional investigation. Multidisciplinary team management by surgeons, gynaecologists, oncologists, pathologists and radiologists is of critical importance. Also, additional large prospective well-designed randomised studies are needed in order to update our current knowledge and provide guidelines to improve the management of patients with EOC.
\end{abstract}

Keywords: Ovarian cancer; cytoreductive surgery; hyperthermic intraperitoneal chemotherapy (HIPEC); chemotherapy

Submitted Feb 27, 2020. Accepted for publication Aug 27, 2020.

doi: 10.21037/jgo-20-130

View this article at: http://dx.doi.org/10.21037/jgo-20-130

\section{Introduction}

Despite the emerging data supporting the beneficial effect of hyperthermic intraperitoneal chemotherapy (HIPEC) in the management of advanced stage epithelial ovarian cancer (EOC) its implementation as standard practice remains sporadic. The recently published recommendation of European Society for Medical Oncology (ESMO) and European Society of Gynecological Oncology (ESGO) consensus on ovarian cancer failed to recommend either intraperitoneal (ip) chemotherapy or HIPEC as a standard of care for the first line management of patients with EOC (level of evidence I and II, respectively and strength of recommendation A for both ip chemotherapy and HIPEC (1). This can be attributed to the lack of welldesigned prospective randomized controlled trials (RCT) for the management of primary advanced stage EOC. In contrast, the latest National Comprehensive Cancer Network (NCCN) guidelines on the management of EOC, 
fallopian tube and primary peritoneal cancer, advocate the use of HIPEC with $100 \mathrm{mg} / \mathrm{m}^{2}$ for stage III disease at the time of interval debulking surgery, followed by systemic intravenous chemotherapy (2). We sought to review the currently available evidence on the use of HIPEC as part of management of primary and recurrent EOC, discuss the importance of selection of the appropriate chemotherapeutic regimen and address directives of future research. We present the following manuscript in accordance with the Narrative Review Reporting Checklist (available at http://dx.doi.org/10.21037/jgo-20-130).

\section{HIPEC for primary EOC}

Two phase III clinical trials are available in the literature evaluating the addition of HIPEC in the management of primary EOC. More specifically, a randomized open-label phase III multicentre trial by Van Driel et al. evaluated the role of HIPEC as part of interval cytoreductive procedures in patients with stage III EOC (M06OVH-OVHIPEC, NCT00426257) (3). All 245 patients received 3 cycles of neoadjuvant chemotherapy with carboplatin (area under the curve of 5 to $6 \mathrm{mg} / \mathrm{mL}$ per minute) and paclitaxel $\left(175 \mathrm{mg} / \mathrm{m}^{2}\right.$ of body-surface area) and were randomly assigned to receive either CRS alone or CRS plus HIPEC through the open technique which was administered by triple dose at $40{ }^{\circ} \mathrm{C}$ for 90 minutes perfusion interval with total cisplatin at $100 \mathrm{mg} / \mathrm{m}^{2}$. After surgery all patients received adjuvant carboplatin and paclitaxel. The authors noted a median overall survival (OS) of 33.9 months for the CRS alone group and 45.7 months for CRS plus HIPEC, whereas the recurrence free survival (RFS) was prolonged by 3.5 months (10.7 months in the CRS group versus 14.2 in the CRS plus HIPEC). Despite the increased rate of major complications in CRS plus HIPEC group compared to CRS alone (27\% vs. $25 \%)$, significance was not reached $(\mathrm{P}=0.76)$. The beneficial effect of HIPEC on survival of patients remained when the patients were further stratified according to certain factors including age, histologic type, previous surgery, number of implants and laparoscopy before surgery.

In contrast, in a second RCT by Lim et al. which was presented at the annual meeting of the American Society of Clinical Oncology in 2017, neither median OS nor median RFS were different (54 vs. 51 months, $\mathrm{P}=0.407$ and 20 vs. 19 months, $\mathrm{P}=0.137$, respectively) among patients from the neoadjuvant subgroup who were randomized to receive either surgery plus HIPEC or no HIPEC for the management of stage III and IV EOC
(NCT01091636) (4). The same was also observed for the overall cohort with comparable 5-year OS and 5-year RFS ( $51 \%$ vs. $49.4 \%, \mathrm{P}=0.574$ and $20.9 \%$ vs. $16 \%, \mathrm{P}=0.569$, respectively). Interestingly, the neoadjuvant chemotherapy (NACT) subgroup after 30 months of OS and 20 months of RFS showed a trend of improved survival in favour of HIPEC which necessitates further long-term observation. Concerning complications, the HIPEC group showed significantly increased rates of anaemia and creatinine elevation compared to control group $(67.4 \%$ vs. $50 \%$, $\mathrm{P}=0.025$ and $15.2 \%$ vs. $4.3 \%, \mathrm{P}=0.026$, respectively). The dose of the intraperitoneal cisplatin was de-escalated to $75 \mathrm{mg}$ which was administered for 90 minutes at $41.5^{\circ} \mathrm{C}$.

It is obvious that further well-designed prospective RCTs are warranted to clarify the role of HIPEC application in the management of primary EOC. For instance, the CHORINE study (NCT01628380) focused on the upfront management of patients with stage IIIC unresectable EOC and tubal cancer with partial or complete response after $1^{\text {st }}$ line NACT ( 3 cycles carboplatin-paclitaxel). After adequate cytoreduction (residual disease $\leq 2.5 \mathrm{~mm}$ ) patients were randomized to either receive HIPEC with cisplatin $100 \mathrm{mg} / \mathrm{m}^{2}$ of body surface area and paclitaxel $175 \mathrm{mg} / \mathrm{m}^{2}$ of body surface area for 90 minutes at $42^{\circ} \mathrm{C}(5,6)$. The estimated completion date has been reached. The outcome of this study as well as of some other ongoing Phase III clinical trials are awaited (7). Finally, the protocol by Batista et al. (NCT02249013) evaluated the efficacy of the "all-in-one approach" for the management of advanced ovarian cancer with NACT followed by fast track CRS and short-course HIPEC with cisplatin $\left(25 \mathrm{mg} / \mathrm{L}\right.$ of perfusate $/ \mathrm{m}^{2}$, with a total limit of $240 \mathrm{mg}$ ) for 30 minutes. The interim analysis of the outcomes demonstrated promising preliminary results (8).

The outcomes of the meta-analysis by Kim et al for patients with primary disease showed an improved diseasefree survival (DFS) in patients who received HIPEC as part of CRS (5 studies, 630 patients, HR: 0.580, 95\% CI: 0.476-0.706) when compared to no-HIPEC. Improved survival remained after subgroup analysis of study design, stage and adjustment for potential confounders (age, stage, NACT, grade, ECOG status and histology) (9). Additionally, the HIPEC groups showed improved OS compared to controls (5 studies, 591 patients, HR: 0.611, 95\% CI: 0.376-0.992). The aforementioned outcomes in favour of the use of HIPEC were also observed in the meta-analysis by Zhang et al. with regards to DFS and OS (HR: $0.41,95 \%$ CI: $0.32-0.54$ and HR: $0.59,95 \%$ CI: $0.46-0.72$, respectively) (10). 
Table 1 Survival outcomes of 190 patients with residual or recurrent ovarian cancer patients reported by Spiliotis et al. (13)

\begin{tabular}{lcccccc}
\hline & \multicolumn{3}{c}{ Residual disease } & & \multicolumn{2}{c}{ Recurrent disease } \\
\cline { 2 - 3 } \cline { 5 - 6 } & CRS + HIPEC + systemic CMT & CRS + systemic CMT & & CRS + HIPEC systemic CMT & CRS + systemic CMT \\
\hline Median OS (months) & 38 & 23.8 & 7 & 26 & 16 \\
Morbidity (\%) & 18 & 1.3 & 3.3 & 15 \\
Mortality (\%) & 2.5 & & 22 & 0 \\
\hline
\end{tabular}

CRS, cytoreductive surgery; HIPEC, hyperthermic intraperitoneal chemotherapy; CMT, chemotherapy.

\section{HIPEC for recurrent EOC}

The use of HIPEC for the secondary management of relapse due to advanced EOC has been more extensively investigated. The first RCT in the field was published by Spiliotis et al. and was highly criticised due to methodological issues $(11,12)$. The authors included 120 patients with advanced stage EOC $(\geq \mathrm{IIIC})$ who had disease recurrence. Patients were randomized to either receive CRS plus HIPEC followed by systemic chemotherapy or CRS with systemic chemotherapy alone. The regimens used for intraperitoneal administration were as follows: for the 34 patients who had platinum-sensitive disease cisplatin $100 \mathrm{mg} / \mathrm{m}^{2}$ and paclitaxel $175 \mathrm{mg} / \mathrm{m}^{2}$ were administered for $60 \mathrm{~min}$ at $42.5^{\circ} \mathrm{C}$ whereas for platinum resistant disease (26 patients) doxorubicin $35 \mathrm{mg} / \mathrm{m}^{2}$ and paclitaxel $175 \mathrm{mg} / \mathrm{m}^{2}$ or mitomycin $15 \mathrm{mg} / \mathrm{m}^{2}$ were administered for $60 \mathrm{~min}$ at $42.5^{\circ} \mathrm{C}$. In 40 patients, an open HIPEC technique was applied, whereas the closed technique was used in the remaining 20 patients. A significant improvement in the mean OS $(\mathrm{P}<0.006)$ and in the 3 -year OS $(\mathrm{P}<0.01)$ was in favour of the HIPEC group. When the authors further compare the outcomes according to platinum responsiveness, no difference was noted among the survival of patients with platinum resistant and platinum sensitive disease either for those with stage IIIC or for those with stage IV. The patients in this protocol are still followed by our study group based on the platinum resistance, including cisplatin plus paclitaxel in platinum sensitive disease and doxorubicin plus paclitaxel or mitomycin in platinum resistant disease. In another study by Spiliotis et al., the authors searched for differences in survival of patients who received cytoreductive surgery (CRS) plus HIPEC as secondary management of residual versus recurrent disease after primary surgery (13). More specifically, 200 patients with relapse of ovarian cancer were divided into 5 groups. Ten patients with splanchnic metastasis received systemic chemotherapy only.
For the 50 patients with recurrent disease, 30 received CRS + HIPEC + systemic chemotherapy and the remaining 20 received CRS and systemic chemotherapy without HIPEC. For the 140 patients with residual disease, 80 had CRS + HIPEC + systemic chemotherapy and the other 60 had CRS and systemic chemotherapy without HIPEC. Table 1 presents the survival outcomes of the study by Spiliotis et al. (10 patients with splanchnic metastases not included) (13). The data suggests a significance of a complete cytoreduction (patients with residual disease) in the primary management of advanced ovarian cancer, which was further enhanced by the addition of HIPEC with improved survival in patients with residual disease compared to recurrent ones (38 vs. 26 months, respectively).

The effect of completeness of cytoreduction in survival of patients has been extensively studied during the last decades with a meta-analysis by Bristow et al. They observed a $5.5 \%$ prolongation in median survival for every $10 \%$ improvement of complete cytoreduction (14). More recently the recommendations regarding goals for the extent of cytoreduction have been changed. No longer is the desired goal the achievement of residual disease $<1 \mathrm{~cm}$. Now the definition of optimal cytoreduction is no macroscopic residual disease (15). Further significant indicators of long-term survival of patients after CRS and HIPEC for advanced EOC are a low Peritoneal Carcinomatosis Index (PCI $\leq 8)$, patients age $\geq 50$ years as well as lower CA-125 levels (CA-125<100). These data were provided by a univariate analysis by Le Saux et al. (16).

The current literature lacks additional RCTs that evaluate the role of HIPEC in the management of disease relapse. However, some ongoing trials have been activated. In that setting, the CHIPOR phase III RCT (NCT01376752) aims to investigate the benefits of CRS and HIPEC in patients with relapse due to ovarian cancer who did not respond to $2^{\text {nd }}$ line platinum-based chemotherapy but have disease that is considered resectable. A total of 444 patients are anticipated to be included based on power analysis and 
are randomised to either receive maximal cytoreduction without HIPEC or maximal cytoreduction plus HIPEC with cisplatin $75 \mathrm{mg} / \mathrm{m}^{2}$ (17). Another RCT actively recruiting is designed to detect the effect of paclitaxel as the chemotherapeutic agent in HIPEC (hipecova NCT02681432). Patients with primary EOC FIGO $\geq$ II or recurrent disease will be randomized to receive CRS plus HIPEC with paclitaxel $\left(175 \mathrm{mg} / \mathrm{m}^{2}\right.$ for 60 minutes at $42-43{ }^{\circ} \mathrm{C}$ ) followed by 6 cycles of systemic intravenous carboplatin $(\mathrm{AUC}=6)$ and paclitaxel $\left(175 \mathrm{mg} / \mathrm{m}^{2}\right)$ compared to CRS and intravenous chemotherapy without HIPEC (18). The meta-analysis by Kim et al. showed patients with recurrent disease due to EOC had a significantly improved OS (7 studies, 491 patients, HR: 0.566, 95\% CI: 0.3790.844) if they received HIPEC compared to those who had a cytoreductive procedure without HIPEC (9). This improved survival remained in the sub-analysis of patients with platinum-sensitive recurrent disease ( 5 studies, HR: 0.616, 95\% CI: 0.402-0.945) (9). However, a difference was not observed in DFS between the two groups (5 studies, 357 patients, HR: 0.644, 95\% CI: 0.395-1.049) (9). Finally, the optimal management in case of recurrent disease still remains a matter of controversy. The addition of chemotherapy and the choice of the appropriate chemotherapeutic regimen is based on the response of the primary disease (platinum-sensitive or platinum-resistant disease). Implementation of secondary CRS will be an option in selected patients especially at the time of first recurrence (19). Recently, Spiliotis et al. highlighted the importance of preoperative administration of 4 cycles of $2^{\text {nd }}$ line systemic chemotherapy (carboplatin and paclitaxel) in platinum-sensitive patients with recurrent EOC who are candidates for CRS and HIPEC (20).

Currently, HIPEC is not considered as the standard of care in the management of either primary or recurrent EOC. Yet, it presents superior outcomes in selected clinical settings as demonstrated by RCTs. According to a survey conducted by the study group of Spiliotis et al and based on questionnaires answered by 467 Medical, Surgical and Gynecologic oncologists, the proportion of physicians who considered there is a utility of HIPEC in the management of primary and recurrent EOC was approximately $50 \%$ and $70 \%$, respectively $(21)$.

\section{Selection of the appropriate chemotherapeutic regimen for HIPEC}

A critical question regards the optimal chemotherapeutic regimen as well as the ideal dose of the agent. There is a significant variety of drugs and drug combinations reported in the literature that are used for HIPEC. As presented above, the most common chemotherapeutic regimens either as upfront or as secondary disease relapse therapy for HIPEC include cisplatin in doses ranging from 75 to $100 \mathrm{mg} / \mathrm{m}^{2}$, paclitaxel $175 \mathrm{mg} / \mathrm{m}^{2}$ alone or cisplatin plus paclitaxel. These regimens are suggested for patients with platinum-sensitive disease. Favourable outcomes have been also reported with administration of $35 \mathrm{mg} / \mathrm{m}^{2}$ doxorubicin and $175 \mathrm{mg} / \mathrm{m}^{2}$ paclitaxel or $15 \mathrm{mg} / \mathrm{m}^{2}$ mitomycin for platinum-resistant disease (11). As mentioned above, to date only cisplatin $100 \mathrm{mg} / \mathrm{m}^{2}$ has been included in the NCCN guidelines as a chemotherapeutic regimen for HIPEC in stage III disease after neoadjuvant chemotherapy (2). Nonetheless, the decisions regarding the preoperative, the postoperative and HIPEC chemotherapeutic regimens is not restricted to the NCCN recommendations. Other combinations of agents such as a paclitaxel containing HIPEC regimen are yet to be examined by randomized trials.

The beneficial effects of HIPEC are based on the supplementary effect of hyperthermia on the pharmacokinetic and pharmacodynamic aspects of cytostatic agents (8). The cytostatic role of hyperthermia and the effect of heat in the improvement of prognosis of patients with various malignancies was studied decades ago. Furthermore, the addition of chemotherapeutic regimens such as cisplatin, bleomycin, mitomycin C and carboplatin, has been proposed to have a significant improvement of survival rates $(22,23)$. More specifically, in ovarian cancer cells treated with cisplatin combined with hyperthermia, an elevation in the intracellular concentration of the agent has been detected as well as a blockage in DNA repair $(22,24)$. In addition to this, the outcomes seem promising in cisplatin resistant cases with the addition of heat (22). A synergic effect of cisplatin and hyperthermia is extensively reported. In contrast, an association has not been established with the use of paclitaxel and heat (25).

It is obvious that there is a significant heterogeneity in the HIPEC protocols. Knowledgeable comparisons among the various treatment regimens to designate the most appropriate protocol is currently not possible. However, these comparisons must be a high priority for future RCTs.

There are a few studies in the literature which compare different chemotherapeutic regimens used for HIPEC. Manzanedo et al. compared 22 patients with advanced stage EOC who received HIPEC with paclitaxel $60 \mathrm{mg} / \mathrm{m}^{2}$ 
body surface area for 60 minutes with 19 others who received cisplatin $100 \mathrm{mg} / \mathrm{m}^{2}$ plus doxorubicin $15 \mathrm{mg} / \mathrm{m}^{2}$ for 90 minutes (25). No difference in DFS and OS among the two groups who received different chemotherapeutic regimens was detected (25). The same lack of difference was observed in a retrospective study by CascalesCampos et al. who evaluated the survival outcomes of patients with advanced EOC (26). Sixty patients received paclitaxel $60 \mathrm{mg} / \mathrm{m}^{2}$ while 51 patients received cisplatin $75 \mathrm{mg} / \mathrm{m}^{2}$ administered at $42-43{ }^{\circ} \mathrm{C}$ for 60 minutes (26). However, the outcomes of these studies are based on a retrospective evaluation of survival. Conclusions for firm decisions warrant further prospective randomized trials to decipher the indications for the use of each regimen. Moreover, new chemotherapeutic agents are also under investigation with the intent to improve the therapeutic effect of intraperitoneal chemotherapy. For instance, dioxadet is an alkylating agent which has been proposed to be suited for intraperitoneal use under hyperthermic effect with promising cytotoxic outcomes as indicated in the experimental protocol by Bespalov et al. $(27,28)$.

\section{Conclusions}

The progress in cytoreductive surgical procedures and chemotherapy has brought significant improvement in the management and survival outcomes of selected patients with advanced EOC. Furthermore, the addition of HIPEC seems encouraging based on the outcomes of high-quality clinical trials. Critical parameters on the use of CRS and HIPEC such as patients' selection, sequencing of procedures, type of chemotherapeutic agent and time and temperature of hyperthermic procedures require additional investigation. Multidisciplinary team management by surgeons, gynaecologists, oncologists, pathologists and radiologists is of great importance and could help to overcome the former limitations. Further prospective well-designed randomised studies are warranted in order to update our current knowledge and guidelines for the treatment of these patients.

\section{Acknowledgments}

Funding: None.

\section{Footnote}

Provenance and Peer Review: This article was commissioned by the Guest Editors (Paul H. Sugarbaker and Kurt van der Speeten) for the focused issue "Intraperitoneal Chemotherapy for Peritoneal Metastases: HIPEC, EPIC, NIPEC, PIPAC and More" published in fournal of Gastrointestinal Oncology. This article has undergone external peer review.

Reporting Checklist: The authors have completed the Narrative Review Checklist. Available at http://dx.doi. org/10.21037/jgo-20-130

Conflicts of Interest: Both authors have completed the ICMJE uniform disclosure form (available at http://dx.doi. org/10.21037/jgo-20-130). The focused issue was sponsored by the Peritoneal Surface Oncology Group International (PSOGI). The authors have no other conflicts of interest to declare.

Ethical Statement: The authors are accountable for all aspects of the work in ensuring that questions related to the accuracy or integrity of any part of the work are appropriately inves tigated and resolved.

Open Access Statement: This is an Open Access article distributed in accordance with the Creative Commons Attribution-NonCommercial-NoDerivs 4.0 International License (CC BY-NC-ND 4.0), which permits the noncommercial replication and distribution of the article with the strict proviso that no changes or edits are made and the original work is properly cited (including links to both the formal publication through the relevant DOI and the license). See: https://creativecommons.org/licenses/by-nc-nd/4.0/.

\section{References}

1. Colombo N, Sessa C, du Bois A, Ledermann J, McCluggage WG, McNeish I, et al. ESMO-ESGO consensus conference recommendations on ovarian cancer: pathology and molecular biology, early and advanced stages, borderline tumours and recurrent disease. Ann Oncol 2019;30:672-705.

2. Armstrong DK, Alvarez RD, Bakkum-Gamez JN, et al. NCCN Guidelines Insights: Ovarian Cancer, Version 1. 2019. J Natl Compr Canc Netw 2019;17:896-909.

3. van Driel WJ, Koole SN, Sikorska K, et al. Hyperthermic Intraperitoneal Chemotherapy in Ovarian Cancer. N Engl J Med 2018;378:230-40.

4. Lim MC, Chang SJ, Yoo HJ, et al. Randomized trial of 
hyperthermic intraperitoneal chemotherapy (HIPEC) in women with primary advanced peritoneal, ovarian, and tubal cancer. J Clin Oncol 2017;35:5520.

5. Ansaloni L, De Iaco P, Frigerio L. Re: "cytoreductive surgery and hyperthermic intraperitoneal chemotherapy as upfront therapy for advanced epithelial ovarian cancer: multi-institutional phase II trial." - Proposal of a clinical trial of cytoreductive surgery and hyperthermic intraperitoneal chemotherapy in advanced ovarian cancer, the CHORINE study. Gynecol Oncol 2012;125:279-81.

6. XXIII AOPG. Phase 3 trial evaluating hyperthermic intraperitoneal chemotherapy in upfront treatment of stage IIIC epithelial ovarian cancer (CHORINE). Clinical Trials gov [Internet]. Bethesda (MD): National Library of Medicine (US), 2000.

7. Naumann RW, Coleman RL, Brown J, et al. Phase III trials in ovarian cancer: The evolving landscape of front line therapy. Gynecol Oncol 2019;153:436-44.

8. Batista TP, Carneiro VCG, Tancredi R, et al. Neoadjuvant chemotherapy followed by fast-track cytoreductive surgery plus short-course hyperthermic intraperitoneal chemotherapy (HIPEC) in advanced ovarian cancer: preliminary results of a promising all-in-one approach. Cancer Manag Res 2017;9:869-78.

9. Kim SI, Cho J, Lee EJ, et al. Selection of patients with ovarian cancer who may show survival benefit from hyperthermic intraperitoneal chemotherapy: A systematic review and meta-analysis. Medicine 2019;98:e18355.

10. Zhang G, Zhu Y, Liu C, et al. The prognosis impact of hyperthermic intraperitoneal chemotherapy (HIPEC) plus cytoreductive surgery (CRS) in advanced ovarian cancer: the meta-analysis. J Ovarian Res 2019;12:33.

11. Spiliotis J, Halkia E, Lianos E, et al. Cytoreductive surgery and HIPEC in recurrent epithelial ovarian cancer: a prospective randomized phase III study. Ann Surg Oncol 2015;22:1570-5.

12. Batista TP. Comment on: Surgery and HIPEC in Recurrent Epithelial Ovarian Cancer: A Prospective Randomized Phase III Study. Ann Surg Oncol 2017;24:630.

13. Spiliotis JD, Iavazzo C, Kopanakis ND, et al. Secondary debulking for ovarian carcinoma relapse: The R-R dilemma - is the prognosis different for residual or recurrent disease? J Turk Ger Gynecol Assoc 2019;20:213-7.

14. Bristow RE, Tomacruz RS, Armstrong DK, et al. Survival effect of maximal cytoreductive surgery for advanced ovarian carcinoma during the platinum era: a metaanalysis. J Clin Oncol 2002;20:1248-59.
15. Liberale G, Pop CF, Polastro L, et al. A radical approach to achieve complete cytoreductive surgery improve survival of patients with advanced ovarian cancer. J Visc Surg 2020;157:79-86.

16. Le Saux O, Decullier E, Freyer G, et al. Long-term survival in patients with epithelial ovarian cancer following cytoreductive surgery and hyperthermic intraperitoneal chemotherapy (HIPEC). Int J Hyperthermia 2018;35:652-7.

17. Classe J. Hyperthermic intra-peritoneal chemotherapy (HIPEC) in relapse ovarian cancer treatment (CHIPOR). ClinicalTrials gov Identifier: NCT01376752.

18. Campos P. Hyperthermic intraperitoneal chemotherapy with paclitaxel in advanced ovarian cancer (hipecova). ClinicalTrials gov Identifier: NCT02681432.

19. Buechel M, Herzog TJ, Westin SN, et al. Treatment of patients with recurrent epithelial ovarian cancer for whom platinum is still an option. Ann Oncol 2019;30:721-32.

20. Spiliotis J, Prodromidou A, Terra A, et al. Recurrent Ovarian Cancer: The role of 2 nd line systemic chemothterapy in combination with cytoreductive surgery plus HIPEC, a retrospective analysis. JBUON 2020. in press.

21. Iavazzo C, Fotiou A, Tsiatas M, et al. Survey on the current practice in the management of advanced ovarian cancer patients-The utility of HIPEC. Pleura Peritoneum 2020;5:20190029.

22. Takahashi I, Emi Y, Hasuda S, et al. Clinical application of hyperthermia combined with anticancer drugs for the treatment of solid tumors. Surgery 2002;131:S78-84.

23. Elias DM, Ouellet JF. Intraperitoneal chemohyperthermia: rationale, technique, indications, and results. Surg Oncol Clin N Am 2001;10:915-33.

24. Cesna V, Sukovas A, Jasukaitiene A, et al. Narrow line between benefit and harm: Additivity of hyperthermia to cisplatin cytotoxicity in different gastrointestinal cancer cells. World J Gastroenterol 2018;24:1072-83.

25. Manzanedo I, Pereira F, Serrano Á, et al. The use of cisplatin plus doxorubicin or paclitaxel in hyperthermic intraperitoneal chemotherapy (HIPEC) for stage IIIC or IV epithelial ovarian cancer: a comparative study. Clin Transl Oncol 2019;21:1357-63.

26. Cascales-Campos P, Lopez-Lopez V, Gil J, et al. Hyperthermic intraperitoneal chemotherapy with paclitaxel or cisplatin in patients with stage III-C/IV ovarian cancer. Is there any difference? Surg Oncol 2016;25:164-70.

27. Bespalov VG, Kireeva GS, Belyaeva OA, et al. Both heat and new chemotherapeutic drug dioxadet in hyperthermic intraperitoneal chemoperfusion improved survival in rat 
ovarian cancer model. J Surg Oncol 2016;113:438-42.

28. Bespalov VG, Alvovsky IK, Tochilnikov GV, et al.

Comparative efficacy evaluation of catheter intraperitoneal chemotherapy, normothermic and hyperthermic chemoperfusion in a rat model of ascitic ovarian cancer. Int J Hyperthermia 2018;34:545-50.

Cite this article as: Spiliotis J, Prodromidou A. Narrative review of hyperthermic intraperitoneal chemotherapy (HIPEC) in patients with advanced ovarian cancer: a critical reappraisal of the current evidence. J Gastrointest Oncol 2021;12 (Suppl 1):S182-S188. doi: 10.21037/jgo-20-130 